\title{
Temática das dissertações e teses em ciência da informação no Programa de Pós-Graduação em Ciências da Comunicação da USP
}

\section{Fernanda Mendes Queiroz}

Bacharel em biblioteconomia pela ECA/USP. Colaboradora do Núcleo de Produção Científica CBD/ECA/USP

E-mail: femendesq@yahoo.com.br

Daisy Pires Noronha

Doutorado em saúde pública

E-mail: daisynor@usp.br

\section{Resumo}

Estudo de natureza descritiva cujo objetivo foi traçar um panorama temático das dissertações de mestrado e teses de doutorado apresentadas ao Programa de Pós-graduação em Ciências da Comunicação - Área de concentração: Ciência da Informação e Documentação - da Escola de Comunicações e Artes da Universidade de São Paulo, no período de 1979 a 2002. O universo de estudo constituiuse de 114 documentos (75 dissertações de mestrado e 39 teses de doutorado). As variáveis da produção analisada referiram-se à distribuição temporal, temáticas abordadas e sua relação com as linhas de pesquisa departamentais. As dissertações/teses foram agrupadas nas categorias da Lista de Cabeçalho de Assunto adotada pelo Library and Information Science Abstracts (LISA). Observou-se que a produção discente da pós-graduação manteve-se estável no período, com tendência a aumentar. Na análise temática, as categorias 19.0 Other Fringe Subjects [Outros Assuntos Correlatos] e 12.0 Bibliographc Records [Registro Bibliográfico] foram as mais abordadas, detendo respectivamente, $22,81 \%$ e $15,54 \%$ da produção. Foram identificados como temas de maior interesse para a elaboração dos trabalhos: ação cultural, sistemas e linguagens de indexação, materiais em C\&T e medicina, bibliotecas públicas e meios de comunicação de massa. Contatou-se que as temáticas abordadas na produção refletem as características da área de concentração e das linhas de pesquisa.

\section{Palavras-chave}

Produção científica; Teses; Dissertações. Ciência da Informação.

Thematic of the master theses and doctoral dissertations in the information science of the Communication Sciences Graduate Program of the University of S. Paulo

\section{Abstract \\ The objective of this study of descriptive nature is to outline an overall view of Master Degree dissertations and Doctorate theses submitted to the Postgraduate Program in Sciences of Communication, mainly in the following areas: Information and Documentation Science, School of Communication and Arts, São Paulo University, from 1979 to 2002. The universe of study comprises 114 documents (75 Master Degree dissertations and 39 Doctorate theses). The variables of the production analized refer to time distribution, thematics approached and their relation to the department lines of research. The dissertations/theses were grouped into categories of the List of Subject Heading, adopted by Library and Information Science Abstracts (LISA). The production was steady during this period. The categories 19.0 Other Fringe Subjects and 12.0 Bibliographic Reccords were the most studied ones, having, respectively, $22.81 \%$ and $15.54 \%$ of production. The themes of greater interest were cultural action, indexing systems and language, subjects on Science and Technology and medicine, public libraries and means of mass communication. \\ Keywords}

Scientific production; Information Science; Dissertations; Thesis.

\section{INTRODUCุÃO}

A ciência é um processo social que tem como funções primordiais disseminar informações, assegurar a preservação de padrões e atribuir crédito e reconhecimento àqueles cujas descobertas têm contribuído para o desenvolvimento do conhecimento (Macias-Chapula, 1998). Sendo assim, ao produzir novos conhecimentos, os pesquisadores devem informar à comunidade científica e posteriormente à sociedade como um todo os seus achados.

O avanço da ciência da informação e das ciências em geral se dá pela constante elaboração de novas pesquisas e pela concretização e divulgação de seus resultados que se processam em diferentes tipos de suportes. A realização dessas pesquisas, no Brasil, concentra-se, na quase totalidade, nos cursos de pós-graduação de universidades públicas. No entanto, vale ressaltar que a pesquisa e a universidade trilharam, em suas origens, caminhos distintos. Os primeiros vínculos entre ensino e pesquisa, mesmo que incipientes, foram observados com o surgimento dos primeiros institutos de pesquisa no país ligados às áreas da botânica e da saúde. A universidade brasileira, no entanto, somente passa a contar com o tripé de sustentação baseado no ensino, pesquisa e extensão com a institucionalização dos cursos de pós-graduação, a partir da reforma universitária de 1968.

Gracelli \& Castro (1985) distinguem, a partir da segunda metade do século XX, três grandes ciclos da pós-graduação brasileira: o primeiro, nas décadas de 50 e 60, que enfatizava a formação de corpo docente; o segundo, na década de 70, com a institucionalização dos cursos de pós-graduação; o terceiro a partir da década de 80 , quando ocorreu a consolidação dos cursos e o desenvolvimento maciço da pesquisa na pós-graduação.

\footnotetext{
* Texto elaborado a partir de trabalho de conclusão de curso apresentado ao CBD/ECA/USP em dez./2003.
} 


\section{Pós-graduação em ciência da informação}

Os primeiros esforços para a implantação da pósgraduação na área da ciência da informação ocorreram em meados da década de $50 \mathrm{com}$ o primeiro curso de pós-graduação lato sensu na área criado pelo Instituto Brasileiro de Bibliografia e Documentação (IBBD).

A implantação da pós-graduação stricto sensu na área se deu, efetivamente, com a criação, em 1970, do primeiro curso de mestrado em ciência da informação pelo IBBD, em convênio com a Universidade Federal do Rio de Janeiro (UFRJ). Atualmente, o Instituto Brasileiro de Informação em Ciência e Tecnologia (IBICT) responde pelo Programa de Pós-Graduação em Ciência da Informação, Mestrado e Doutorado junto à Universidade Federal Fluminense (UFF). Esses institutos atuam há quase meio século na titulação de profissionais na área oriundos de todo o Brasil. A pós-graduação, em nível de doutorado, tem como marco inicial a criação, em 1980, pela Escola de Comunicações e Artes da Universidade de São Paulo (ECA/USP), da área de concentração em ciência da informação junto ao Programa de PósGraduação em Ciências da Comunicação. Destaca-se que o primeiro programa de doutorado específico na área foi criado em 1992 na Universidade de Brasília (UnB). Os principais fatores que influenciaram a implantação da pós-graduação na área recaem nas necessidades da formação de recursos humanos especializados, na capacitação de docentes para as instituições de ensino superior e na conscientização da importância do desenvolvimento da pesquisa em informação (Población, 1993).

Atualmente existem no Brasil oito cursos de pós-graduação em ciência da informação, sendo sete programas e uma área de concentração (dentro do Programa de Ciências da Comunicação) credenciados pela Capes (Núcleo de Produção Científica).

Pós-graduação na ECA/USP: Programa de Pósgraduação em Ciências da Comunicação - Área de concentração: Ciência da Informação e Documentação

A Escola de Comunicações Culturais da Universidade de São Paulo (ECC/USP) foi criada em 1966, dando início às atividades em 1967 com os cursos de graduação nas áreas de jornalismo, rádio e televisão, relações públicas, teatro, cinema, biblioteconomia e documentação.

As atividades da pós-graduação foram iniciadas em 1972 com a implantação do programa de mestrado em ciências da comunicação e, em 1974, do programa de mestrado em artes. $\mathrm{O}$ programa de doutorado em ambas as áreas começou em 1980. As primeiras turmas de pós-graduados vinculadas à área de concentração ciência da informação eram compostas principalmente pelos docentes do Departamento de Biblioteconomia e Documentação (CBD), devido à necessidade de titulação dos mesmos para que pudessem exercer suas atividades de maneira regulamentada na ECA.

Somente em 1986 foram instauradas as linhas de pesquisa com o objetivo de "[....] dar uma configuração mais precisa à temática de investigação dos Departamentos [....] e definir melhor o campo de pesquisa da ECA, preservando a sua diversidade e interdisciplinaridade." (Pinto, 1988, p. 109). Atualmente a área de concentração em ciência da informação e documentação do Programa de Pós-graduação em Ciências da Comunicação da ECA possui quatro linhas de pesquisa: Ação Cultural, Análise Documentária e Geração e Uso da Informação, criadas em 1986; e Informação, Comunicação e Educação, criada em 1998, como desdobramento da linha Ação Cultural. A partir de então, a produção de dissertações/teses começou a ser identificada em função da redefinição das áreas de abrangência temática das linhas, onde se encontram agrupados os docentes/pesquisadores por áreas de interesse.

É nesse contexto que se insere o presente trabalho, que visa a avaliar um seguimento da produção da pósgraduação em ciência da informação representado pela análise da temática das dissertações/teses defendidas no $\mathrm{CBD} / \mathrm{ECA} / \mathrm{USP}$.

A literatura tem mostrado que estudos voltados à análise temática constituem importante "referencial sobre o que vem sendo efetivado em termos de produção científica em uma determinada área do conhecimento." (Witter \& Pécora, 1997, p. 79), detectando-se possíveis modismos da ciência que evidenciam ou não determinados temas.

Macedo (1987) analisou questões relacionadas à pesquisa (dissertações/teses) sob três aspectos: proveniência dos estudos, problemas/obstáculos e temática abordada.

Witter \& Pécora (1997) levantaram os temas enfocados nas dissertações/teses produzidas no Brasil, no período 1970-1992. Concluiu-se que muitos temas da área não são abordados nas pesquisas, mesmo existindo núcleos temáticos, e que as investigações efetuadas nos programas de pós-graduação, quando comparadas, abordam assuntos dispersos. 
Teixeira (1997) analisou as tendências temáticas das dissertações do curso de Mestrado em Biblioteconomia e Documentação da Universidade de Brasília, defendidas entre 1980 e 1995, e as relações da produção como as linhas de pesquisa do curso. Observou-se que a produção discente está de acordo com a proposta do curso em geral.

Oliveira (1999) analisou as dissertações do curso de mestrado em biblioteconomia e documentação da Universidade Federal da Paraíba, relativas ao período de 1981 a 1998, com o objetivo de apreender as características básicas desta produção, como, por exemplo, os temas pesquisados.

Evangelista (2002) identificou os objetos de estudo das dissertações do mestrado em biblioteconomia da PUCCampinas, no período de 1980-2001, com o objetivo de mostrar o que o mestrando pesquisou ao longo da existência do curso, levando em consideração a relação com as linhas de pesquisa. Concluiu-se que o pesquisador na área da informação estuda diversos objetos que podem estar relacionados a assuntos de qualquer área do conhecimento.

Araújo e col. (2003), em estudo com dissertações em ciência da informação da UFPb, apontam como ponto forte a interdisciplinaridade dos alunos e os temas das pesquisas.

\section{OBJETIVOS}

Os objetivos deste trabalho são:

- identificar a distribuição temporal da produção das dissertações de mestrado e teses de doutorado;

- identificar a distribuição das temáticas estudadas nas dissertações de mestrado e teses de doutorado, segundo as linhas de pesquisa.

\section{MÉTODO}

O universo de estudo foi composto por 114 documentos, sendo 75 dissertações de mestrado e 39 teses de doutorado defendidas no Programa de Pós-graduação em Ciências da Comunicação - Área de concentração: Ciência da Informação e Documentação da ECA/USP, no período de 1979 a 2002, identificados no Banco de Dados de Dissertações e Teses da ECA/USP, mantido pelo Serviço de Biblioteca e Documentação dessa escola, que cobre todo o período estudado (1979-2002).

\section{Caracterização das dissertações/teses}

A caracterização das dissertações/teses foi feita a partir da identificação do autor, título, ano, grau (mestrado/ doutorado), orientador e respectiva linha de pesquisa.

A alocação das dissertações/teses nas linhas de pesquisa se deu mediante a identificação do orientador do trabalho e respectivas linhas: Ação Cultural, Análise Documentária, Geração e Uso da Informação e Informação, Comunicação e Educação.

\section{Temática das dissertações/teses}

Para a classificação temática das dissertações/teses, utilizou-se o Broad Subject Headings (Lista de Cabeçalho de Assunto), adotado pelo Library and Information Science Abstracts - Lisa (Library..., 2003) em sua versão impressa* - que é composta por 19 categorias temáticas, subdividas em assuntos específicos. Optou-se por esse instrumento pela sua completeza no campo de atuação da área, além de se constituir em obra de referência de âmbito internacional.

Cada dissertação/tese foi classificada em apenas uma das 19 categorias da lista. Para a especificidade dos assuntos, cada documento foi identificado com uma subcategoria que se enquadra dentro das 19 categorias da Lista de Cabeçalho de Assunto.

Para melhor desenvolvimento deste estudo, os termos do LISA foram mantidos em inglês, acrescidos de tradução literal para o português. Procurou-se, com isso, mesmo sem a utilização de critérios normativos específicos, dar maior amplitude de compreensão ao leitor.

\section{RESULTADOS E DISCUSSÃO}

\section{Distribuição temporal da produção das dissertações/teses}

O universo estudado, representado no gráfico 1 , a seguir, é composto por 75 dissertações de mestrado e 39 teses de doutorado, totalizando 114 trabalhos produzidos no período entre 1979 e 2002, distribuídos segundo ano de conclusão, possibilitando assim a visualização do panorama evolutivo da produção. Observa-se uma trajetória de crescimento tanto para o mestrado quanto para o doutorado, com picos de aumento e queda ocorrendo em períodos semelhantes.

* Em sua versão eletrônica, disponibilizado pelo Banco de Dados WebSPIRIS - Eletronic Reference Library, o LISA utiliza termos estruturados em Tesauro. 
Temática das dissertações e teses em ciência da informação do Programa de Pós-Graduação em Ciências da Comunicação da USP

O aumento pode estar relacionado com o vencimento dos prazos para conclusão dos trabalhos, e as quedas, com a diminuição natural do número de discentes após a formação de um grupo representativo de mestres e doutores. Nota-se, a partir de 1998, um crescimento acelerado da produção, que possivelmente se deva à consolidação da área de concentração e da formação de massa crítica com um corpo docente titulado e credenciado para a orientação de trabalhos em nível de pós-graduação (mestrado e doutorado), o que leva ao aumento do oferecimento de vagas e à expansão da atividade investigativa nas linhas de pesquisa.

Análise temática das dissertações/teses

A tabela 1 apresenta o panorama da distribuição temática das dissertações/teses classificadas nas categorias da Lista de Cabeçalho de Assunto adotada pelo LISA. Observa-se a concentração da produção em 5 das 19 categorias da Lista, identificando assim os eixos temáticos do programa.

A categoria 19.0 Other Fringe Subjects (Outros Assuntos Correlatos), que deteve o maior número da produção com 26 trabalhos (22,81\%), agrupa, em sua maioria, estudos interdisciplinares que agregam à ciência da informação discussões de outras áreas. A tendência da exploração de temas interdisciplinares na pós-graduação da área também foi comentada por Macedo (1987), Oliveira (1999) e Teixeira (1997), mas em nenhum desses estudos
GRÁFICO 1

Distribuição das dissertações/teses segundo ano de conclusão (1979-2002)

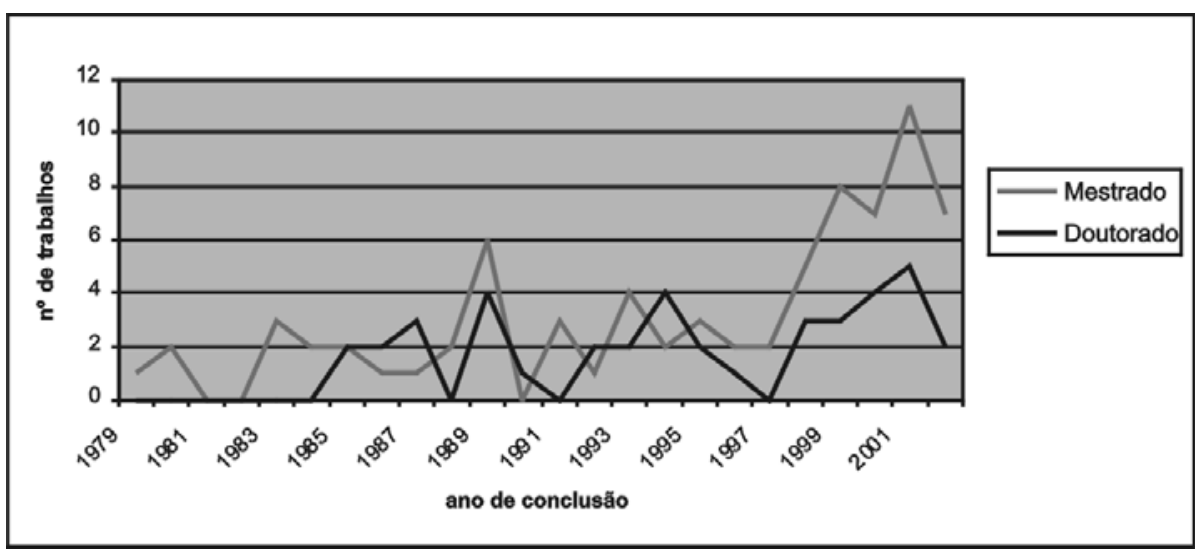

TABELA 1

Distribuição temática das dissertações/teses (1979-2002) “Categorias Temáticas Gerais”

\begin{tabular}{|c|c|c|c|c|}
\hline \multirow{2}{*}{ CATEGORIAS TEMÁTICAS } & \multicolumn{2}{|c|}{ TOTAL } & \multicolumn{2}{|c|}{ TG } \\
\hline & $\mathrm{M}$ & $\mathrm{D}$ & $\mathrm{N}^{\circ}$ & $\%$ \\
\hline 1.0 LIBRARIANSHIP AND INFORMATION SCIENCE [Bibliotec. e Cien. Inf.] & 1 & 1 & 2 & 1,75 \\
\hline 2.0 PROFESSION [Profissöes & 2 & 1 & 3 & 2,63 \\
\hline 3.0 LIBRARIES AND RESOURCE CENTRES [Bibliot. e Centr. de Inf.] & 11 & 7 & 18 & 15,79 \\
\hline 4.0 LIBRARY USE AND USERS [Uso de Bibliotecas e Usuários] & 6 & 6 & 12 & 10,53 \\
\hline 5.0 MATERIALS [Materiais] & 7 & 5 & 12 & 10,53 \\
\hline 6.0 ORGANIZATION [Organizaçao] & 1 & 2 & 3 & 2,63 \\
\hline 7.0 LIBRARY BUILDINGS [Prédios de Bibliotecas] & . & . & . & - \\
\hline 8.0 LIBRARY TECHNOLOGY [Tecnologia em Bibliotecas] & - & 1 & 1 & 0,88 \\
\hline 9.0 TECHNICAL SERVICES [Serviços Técnicos] & 3 & 1 & 4 & 3,51 \\
\hline 10.0 INFORMATION COMMUNICATION [Difusão da Informação] & 2 & - & 2 & 1,75 \\
\hline 11.0 BIBLIOGRAPHIC CONTROL [Controle Bibliográfico] & . & . & . & . \\
\hline 12.0 BIBLIOGRAPHIC RECORDS [Registro Bibliográfico] & 12 & 8 & 20 & 17,54 \\
\hline $\begin{array}{l}\text { 13.0 COMPUTERIZED INFORMATION STORAGE AND RETRIEVAL } \\
\text { [Armaz. e Recuper. Comput. da Inf.] }\end{array}$ & 2 & 2 & 4 & 3,51 \\
\hline $\begin{array}{l}\text { 14.0 COMMUNICATIONS AND INFORMATION TECHNOLOGY [Tec. da } \\
\text { Inf. e Comunicação] }\end{array}$ & 3 & 1 & 4 & 3,51 \\
\hline 15.0 READING [Leitura] & 2 & 1 & 3 & 2,63 \\
\hline 16.0 MEDIA [Mídia] & - & - & - & . \\
\hline 17.0 KNOWLEDGE AND LEARNING [Conhecimento e Aprendizado] & - & - & - & - \\
\hline 18.0 RECORDS MANEGEMENT [Gerenciamento de Registros] & . & - & . & . \\
\hline 19.0 OTHER FRINGE SUBJECTS [Outros Assuntos Correlatos] & 23 & 3 & 26 & 22,81 \\
\hline TOTAL & 75 & 39 & 114 & 100 \\
\hline
\end{tabular}

LEGENDA: D - Doutorado; M - Mestrado; TG - Total Geral 
obtiveram-se números tão expressivos como os da produção aqui analisada. Tal fato possivelmente se deva às características do Programa da ECA/USP, que tem em sua formação docentes e pesquisadores advindos de diferentes áreas humanísticas, e a preocupação dos pós-graduados, nos primeiros anos do curso, em estudar temas relacionados às áreas de comunicação, artes e cultura brasileira.

Relação das temáticas das dissertações/ teses com as linhas de pesquisa

O volume de dissertações/teses concluídas segundo as linhas de pesquisa pode ser visualizado no gráfico 2 .

A incidência de maior número de trabalhos nas linhas Ação Cultural, Análise Documentária e Geração e Uso da Informação está relacionada ao ano da criação das mesmas. Enquanto estas foram criadas em 1986, a linha Informação, Comunicação e Educação foi instituída somente em 1998, com desdobramento da Ação Cultural. Foram categorizadas como Sem Indicação de Linha as dissertações/ teses concluídas antes da criação das mesmas ou aquelas orientadas por docentes não pertencentes ao CBD.

Verificando as temáticas abordadas nas dissertações/teses segundo a linha de pesquisa em que foram elaboradas e concluídas, obtém-se um quadro comparativo entre a produção e o objetivo das linhas.

A produção de dissertações/teses na linha Ação Cultural (tabela 2, a seguir) está concentrada na categoria 19.0 Other Fringe Subjects [Outros Assuntos Correlatos] totalizando $48,65 \%$. Esse resultado era previsível pois a referida linha tem por objetivo pesquisar os diversos modos da política cultural, como política pública e as variáveis da ação cultural (USP, 2002). Logo, apesar da estreita relação com a ciência da informação, a linha não se restringe a ela. As relações da linha Ação Cultural com a área podem ser observadas, por exemplo, em 3.0 Libraries and Resource Centres [Bibliotecas e Centros de Pesquisa], cujos trabalhos abordam aspectos da mesma em bibliotecas públicas, serviços de informação em arte e museus.

Na tabela 3, a seguir, é apresentada a distribuição das temáticas abordadas nas dissertações/teses concluídas na linha Análise Documentária. A concentração da produção (55,55\%) na categoria 12.0 Bibliographc Records [Registros Bibliográficos] justifica-se pelos temas específicos relacionados aos procedimentos e instrumentos de representação e análise documentária. $\bigcirc$ número de trabalhos existentes na categoria 13.0 Computerized Information Storage and Retrieval [Armazena-mento e Recuperação Computadori-zada da Informação] indicam a preocupação da linha com a aplicação da tecnologia na área. A dispersão da produção nas demais categorias pode ser relacionada com o interesse da linha em investigar as "[....] interfaces da análise documen-tária como a lingüística, a lógica, a terminologia e a comunicação." (USP, 2002, p. 29).

A produção obtida na linha Geração e Uso da Informação (tabela 4, a seguir), a seguir, mostra a diversidade de temas que a linha objetiva estudar, relacionados ao fluxo da comunicação da informação. Logo, a concentração de estudos (41,67\%) nas categorias 4.0 Libraries Use and Users [Uso de Bibliotecas e Usuários] e 5.0 Materials [Materiais] representa a preocupação da linha para assuntos relacionados ao comportamento de busca da informação, a análise da produção científica e a identificação das características dos usuários da informação. (USP, 2002).

A linha Informação, Comunicação e Educação apresenta em sua produção uma diversidade temática, como pode ser observado na tabela 5, a seguir. Esse panorama pode ser justificado pela caracterização da linha que enfatiza diferentes aspectos das relações entre informação, comunicação e educação com destaque para as "[....] novas concepções de bibliotecas, processos e serviços de informação e cultura." (USP, 2002, p. 30), como ilustram as categorias 4.0 e 19.0. Ainda é possível relacionar a dispersão de temas com o pequeno número de trabalhos concluídos em detrimento das demais linhas de pesquisa com o fato de ser uma linha criada mais recentemente e ainda não estar completamente consolidada. 
Temática das dissertações e teses em ciência da informação do Programa de Pós-Graduação em Ciências da Comunicação da USP

TABELA 2

Distribuição temática das dissertações/teses segundo linha de pesquisa "Ação Cultural"

\begin{tabular}{|c|c|c|c|}
\hline \multirow{2}{*}{ CATEGORIAS/SUBCATEGORIAS TEMÁTICAS } & \multicolumn{3}{|c|}{ TRAB. CONC. } \\
\hline & $\mathrm{M}$ & $\mathrm{D}$ & $\mathrm{T}$ \\
\hline $\begin{array}{l}1.0 \text { - LIBRARIANSHIP AND INFORMATION SCIENCE [Bibliotec. E Cien. } \\
\text { Inf.] }\end{array}$ & - & 1 & 1 \\
\hline Subtotal & 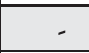 & 1 & 1 \\
\hline $\begin{array}{l}3.0 \text { - LIBRARIES AND RESOURCE CENTRES [Bibliot. e Centr. De Inf.] } \\
\text { libraries and resource centres [bibliotecas e centros de Informação] } \\
\text { public libraries [bibliotecas públicas] } \\
\text { special subject libraries, research libraries [bibliotecas especializadas, centros } \\
\text { de pesquisa] } \\
\text { museums [museus] }\end{array}$ & $\begin{array}{l}1 \\
2\end{array}$ & $\dot{2}$ & $\begin{array}{l}1 \\
4\end{array}$ \\
\hline Subtotal & 6 & 4 & 10 \\
\hline $\begin{array}{l}4.0 \text { - LIBRARY USE AND USERS [Uso de Bibliotecas e Usuários] } \\
\text { libraries and services by types of user [bibliotecas e serviços por tipo de } \\
\text { usuário] } \\
\text { school libraries [bibliotecas escolares] } \\
\text { user training [treinamento de usuário] }\end{array}$ & $\dot{-}$ & $\begin{array}{l}- \\
1 \\
1\end{array}$ & $\begin{array}{l}1 \\
1\end{array}$ \\
\hline Subtotal & 1 & 2 & 3 \\
\hline $\begin{array}{l}9.0 \text { - TECHNICAL SERVICES [Serviços Técnicos] } \\
\text { preservation [preservação] }\end{array}$ & 2 & - & 2 \\
\hline Subtotal & 2 & - & 2 \\
\hline $\begin{array}{l}12.0 \text { - BIBLIOGRAPHIC RECORDS [Registro Bibliográfico] } \\
\text { index language and systems [sistemas e linguagens de indexação] }\end{array}$ & - & 1 & 1 \\
\hline Subtotal & - & 1 & 1 \\
\hline $\begin{array}{l}\text { 14.0 - COMMUNICATIONS AND INFORMATION TECHNOLOGY [Tec. da } \\
\text { Inf. e Com.] } \\
\quad \text { computers [computadores] }\end{array}$ & 1 & - & 1 \\
\hline Subtotal & 1 & - & 1 \\
\hline 15.0 - READING [Leitura] & - & 1 & 1 \\
\hline Subtotal & - & 1 & 1 \\
\hline $\begin{array}{l}\text { 19.0 - OTHER FRINGE SUBJECTS [Outros Assuntos Correlatos] } \\
\text { ação cultural } \\
\text { Arte } \\
\text { Lingüística } \\
\text { meios de comunicação de massa } \\
\text { Música }\end{array}$ & $\begin{array}{l}9 \\
2 \\
- \\
4 \\
1\end{array}$ & $\begin{array}{l}1 \\
- \\
1 \\
-\end{array}$ & $\begin{array}{l}10 \\
2 \\
1 \\
4 \\
1\end{array}$ \\
\hline Subtotal & 16 & 2 & 18 \\
\hline TOTAL & 26 & 11 & 37 \\
\hline
\end{tabular}

LEGENDA: Doutorado; M - Mestrado; T - Total 
TABELA 3

Distribuição temática das dissertações/teses segundo linha de pesquisa "Análise Documentária”

\begin{tabular}{|c|c|c|c|}
\hline \multirow[t]{2}{*}{ CATEGORIAS/SUBCATEGORIAS TEMÁTICAS } & \multicolumn{3}{|c|}{$\begin{array}{l}\text { TRAB. } \\
\text { CONC. }\end{array}$} \\
\hline & $\mathrm{M}$ & $\mathrm{D}$ & $\mathrm{T}$ \\
\hline 1.0 - LIBRARIANSHIP AND INFORMATION SCIENCE [Bibliotec. e Cien. Inf.] & 1 & - & 1 \\
\hline Subtotal & 1 & - & 1 \\
\hline $\begin{array}{l}3.0 \text { - LIBRARIES AND RESOURCE CENTRES [Bibliot. e Centr. de Inf.] } \\
\text { libraries of other organizations and private libraries [bibliot. de outras } \\
\text { organizações e bibliot. privadas] }\end{array}$ & 1 & 1 & 2 \\
\hline Subtotal & 1 & 1 & 2 \\
\hline $\begin{array}{l}4.0 \text { - LIBRARY USE AND USERS [Uso de Bibliotecas e Usuários] } \\
\text { libraries and services by types of user [bibliotecas e serviços por tipo de usuário] }\end{array}$ & 1 & - & 1 \\
\hline Subtotal & 1 & - & 1 \\
\hline $\begin{array}{l}5.0 \text { - MATERIALS [Materiais] } \\
\text { periodicals and newspapers [periódicos e jornais] }\end{array}$ & 1 & - & 1 \\
\hline Subtotal & 1 & - & 1 \\
\hline $\begin{array}{l}6.0 \text { - ORGANIZATION [Organizaçao] } \\
\text { management (other than personnel management) [adm.- outros diferentes de } \\
\text { adimin. de pessoal] }\end{array}$ & - & 1 & 1 \\
\hline Subtotal & - & 1 & 1 \\
\hline $\begin{array}{l}10.0 \text { - INFORMATION COMMUNICATION [Difusão da Informação] } \\
\text { science, technology, medicine information work [trabalhos de informação em } \\
\text { CETT e medicina] }\end{array}$ & 1 & - & 1 \\
\hline Subtotal & 1 & - & 1 \\
\hline $\begin{array}{l}12.0 \text { - BIBLIOGRAPHIC RECORDS [Registro Bibliográfico] } \\
\text { cataloguing and indexing [catalogação e indexação] } \\
\text { indexing [indexação] } \\
\text { index language and systems [sistemas e linguagens de indexação] } \\
\text { thesauri [tesauro] }\end{array}$ & $\begin{array}{l}3 \\
- \\
6 \\
1\end{array}$ & $\begin{array}{l}- \\
3 \\
2\end{array}$ & $\begin{array}{l}3 \\
3 \\
8 \\
1\end{array}$ \\
\hline Subtotal & 10 & 5 & 15 \\
\hline $\begin{array}{l}13.0 \text { - COMPUTERIZED INFORMATION STORAGE AND RETRIEVAL [Armaz. e } \\
\text { Recuper. Comput. da Inf.] } \\
\text { automatic text analysis, automatic indexing, machine translation [anal. } \\
\text { automat. de textos, index., trad.] } \\
\text { databases in general [bases de dados em geral] } \\
\text { online systems [sistemas on-line] }\end{array}$ & $\dot{1}$ & $\begin{array}{l}1 \\
1 \\
\end{array}$ & $\begin{array}{l}1 \\
2 \\
\end{array}$ \\
\hline Subtotal & 2 & 2 & 4 \\
\hline $\begin{array}{l}19.0 \text { - OTHER FRINGE SUBJECTS [Outros Assuntos Correlatos] } \\
\text { meios de comunicação de massa }\end{array}$ & 1 & - & 1 \\
\hline Subtotal & 1 & - & 1 \\
\hline TOTAL & 18 & 9 & 27 \\
\hline
\end{tabular}

LEGENDA: D - Doutorado; M - Mestrado; T - Total 
Temática das dissertações e teses em ciência da informação do Programa de Pós-Graduação em Ciências da Comunicação da USP

TABELA 4

Distribuição temática das dissertações/teses segundo linha de pesquisa "Geração e Uso da Informação"

\begin{tabular}{|c|c|c|c|}
\hline \multirow{2}{*}{ CATEGORIAS/SUBCATEGORIAS TEMÁTICAS } & \multicolumn{3}{|c|}{ TRAB.CONC } \\
\hline & $\mathrm{M}$ & $\mathrm{D}$ & $\mathrm{T}$ \\
\hline $\begin{array}{l}2.0 \text { - PROFESSION [Profissões] } \\
\text { education and training [ensino e treinamento] }\end{array}$ & - & 1 & 1 \\
\hline Subtotal & - & 1 & 1 \\
\hline $\begin{array}{l}3.0 \text { - LIBRARIES AND RESOURCE CENTRES [Bibliot. e Centr. De Inf.] } \\
\text { academic libraries (not school libraries) [bibliot. Acadêmicas - não bibliot. } \\
\text { escolares] }\end{array}$ & 1 & 1 & 2 \\
\hline Subtotal & 1 & 1 & 2 \\
\hline $\begin{array}{l}4.0 \text { - LIBRARY USE AND USERS [Uso de Bibliotecas e Usuários] } \\
\text { libraries and services by types of user [bibliotecas e serviços por tipo de usuário] } \\
\text { school libraries [bibliotecas escolares] } \\
\text { user training [treinamento de usuário] } \\
\text { interloans and photocopying services [emprest. entre bibliot. e serviços de } \\
\text { fotocópia] }\end{array}$ & $\begin{array}{l}1 \\
1\end{array}$ & $\begin{array}{l}2 \\
1 \\
- \\
1\end{array}$ & $\begin{array}{l}3 \\
1 \\
1 \\
1\end{array}$ \\
\hline Subtotal & 2 & 4 & 6 \\
\hline $\begin{array}{l}5.0 \text { - MATERIALS [Materiais] } \\
\text { subjects [assuntos] } \\
\text { humanities materials [materiais em humanidades] } \\
\text { science, technology, medicine materials [materiais em CËT e medicina] }\end{array}$ & $\begin{array}{l}1 \\
- \\
-\end{array}$ & $\begin{array}{l}- \\
1 \\
2\end{array}$ & $\begin{array}{l}1 \\
1 \\
2\end{array}$ \\
\hline Subtotal & 1 & 3 & 4 \\
\hline $\begin{array}{r}6.0 \text { - ORGANIZATION [Organizaçao] } \\
\text { public relations [relações públicas] }\end{array}$ & - & 1 & 1 \\
\hline Subtotal & - & 1 & 1 \\
\hline $\begin{array}{l}8.0 \text { - LIBRARY TECHNOLOGY [Tecnologia em Bibliotecas] } \\
\text { computers [computadores] }\end{array}$ & - & 1 & 1 \\
\hline Subtotal & - & 1 & 1 \\
\hline $\begin{array}{l}9.0 \text { - TECHNICAL SERVICES [Serviços Técnicos] } \\
\text { collection development [desenvolvimento de coleções] }\end{array}$ & - & 1 & 1 \\
\hline Subtotal & - & 1 & 1 \\
\hline $\begin{array}{l}10.0 \text { - INFORMATION COMMUNICATION [Difusão da Informação] } \\
\text { reference work [trabalhos de referência] }\end{array}$ & 1 & - & 1 \\
\hline Subtotal & 1 & - & 1 \\
\hline $\begin{array}{l}12.0 \text { - BIBLIOGRAPHIC RECORDS [Registro Bibliográfico] } \\
\text { cooperative cataloguing, bibliographic utilities [catalog. coop., serviços } \\
\text { bibliográficos] } \\
\text { cataloguing rules [regras de catalogação] } \\
\text { thesauri [tesauro] }\end{array}$ & 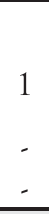 & $\begin{array}{l}- \\
1 \\
1\end{array}$ & $\begin{array}{l}1 \\
1 \\
1\end{array}$ \\
\hline Subtotal & 1 & 2 & 3 \\
\hline $\begin{array}{l}\text { 14.0 - COMMUNICATIONS AND INFORMATION TECHNOLOGY [Tec. da Inf. } \\
\text { e Comunicação] }\end{array}$ & & & \\
\hline networks [redes] & 1 & 1 & 2 \\
\hline software [programas de computador] & 1 & - & 1 \\
\hline Subtotal & 2 & 1 & 3 \\
\hline $\begin{array}{r}15.0 \text { - READING [Leitura] } \\
\text { literacy [alfabetização] }\end{array}$ & 1 & - & 1 \\
\hline Subtotal & 1 & - & 1 \\
\hline TOTAL & 9 & 15 & 24 \\
\hline
\end{tabular}

LEGENDA: D - Doutorado; M - Mestrado; T - Total 
TABELA 5

Distribuição temática das dissertações/teses segundo linha de pesquisa "Informação, Comunicação e Educação"

\begin{tabular}{|c|c|c|c|}
\hline \multirow{2}{*}{ CATEGORIAS/SUBCATEGORIAS TEMÁTICAS } & \multicolumn{3}{|c|}{ TRAB.CONC } \\
\hline & $\mathrm{M}$ & $\mathrm{D}$ & $\mathrm{T}$ \\
\hline $\begin{array}{l}2.0 \text { - PROFESSION [Profissões] } \\
\text { library and information staff [equipes em informação e biblioteca] }\end{array}$ & 2 & - & 2 \\
\hline Subtotal & 2 & - & 2 \\
\hline $\begin{array}{l}3.0 \text { - LIBRARIES AND RESOURCE CENTRES [Bibliot. e Centr. de Inf.] } \\
\text { academic libraries (not school libraries) [bibliotecas acadêmicas - exceto } \\
\text { bibliotecas escolares] }\end{array}$ & 2 & - & 2 \\
\hline Subtotal & 2 & - & 2 \\
\hline $\begin{array}{l}4.0 \text { - LIBRARY USE AND USERS [Uso de Bibliotecas e Usuários] } \\
\text { user services [serviços ao usuário] } \\
\text { document delivery [entrega de documentos] }\end{array}$ & $\begin{array}{l}1 \\
1\end{array}$ & - & $\begin{array}{l}1 \\
1\end{array}$ \\
\hline Subtotal & 2 & - & 2 \\
\hline $\begin{array}{l}5.0 \text { - MATERIALS [Materiais] } \\
\text { science, technology, medicine materials [materiais em ciência, tecnologia e } \\
\text { medicina] }\end{array}$ & - & 1 & 1 \\
\hline Subtotal & - & 1 & 1 \\
\hline $\begin{array}{l}6.0 \text { - ORGANIZATION [Organizaçao] } \\
\text { management (other than personnel management) }[\text { adm. - exceto adm. de } \\
\text { pessoal }]\end{array}$ & 1 & - & 1 \\
\hline Subtotal & 1 & - & 1 \\
\hline $\begin{array}{l}9.0 \text { - TECHNICAL SERVICES [Serviços Técnicos] } \\
\text { collection development [desenvolvimento de coleções] }\end{array}$ & 1 & - & 1 \\
\hline Subtotal & 1 & - & 1 \\
\hline $\begin{array}{l}\text { 19.0 - OTHER FRINGE SUBJECTS [Outros Assuntos Correlatos] } \\
\text { ação cultural } \\
\text { Literatura } \\
\text { narrativa oral }\end{array}$ & $\begin{array}{l}1 \\
2 \\
1\end{array}$ & - & $\begin{array}{l}1 \\
2 \\
1\end{array}$ \\
\hline Subtotal & 4 & - & 4 \\
\hline TOTAL & 12 & 1 & 13 \\
\hline
\end{tabular}

LEGENDA: CAT - D - Doutorado; M - Mestrado; T - Total

Os trabalhos identificados como Sem Indicação de Linha referem-se aos primeiros anos do curso. Como não existiam linhas de pesquisa e áreas de concentração, esperava-se maior diversidade de temáticas principalmente aquelas classificadas na categoria 19.0 Other Fringe Subjects [Outros Assuntos Correlatos]. Porém, verifica-se, na tabela 6 , a seguir, que as temáticas abordadas sugerem os primeiros delineamentos para a formação das linhas.
Considerando-se os temas específicos, sem se levar em conta as categorias agrupadas nas linhas de pesquisa, verifica-se que o maior interesse para a elaboração dos trabalhos recai sobre os temas ação cultural (11), sistemas e linguagens de indexação (10), materiais em CËT e medicina (7), bibliotecas públicas (6) e meios de comunicação de massa (6), estudados por $35,09 \%$ das dissertações/teses concluídas. Ação cultural, meios de comunicação de massa e sistemas e linguagens de indexação predominaram nas dissertações de mestrado e materiais em CËT e medicina nas teses de doutorado; bibliotecas públicas foi de igual interesse em ambas as categorias. 
Temática das dissertações e teses em ciência da informação do Programa de Pós-Graduação em Ciências da Comunicação da USP

TABELA 6

Distribuição temática das dissertações/teses segundo linha de pesquisa "Sem Indicação de Linha"

\begin{tabular}{|c|c|c|c|}
\hline \multirow{2}{*}{ CATEGORIAS/SUBCATEGORIAS TEMÁTICAS } & \multicolumn{3}{|c|}{ TRAB.CONC } \\
\hline & $\mathrm{M}$ & D & $\mathrm{T}$ \\
\hline $\begin{array}{l}3.0 \text { - LIBRARIES AND RESOURCE CENTRES [Bibliot. e Centr. de Inf.] } \\
\text { public libraries [bibliotecas públicas] }\end{array}$ & 1 & 1 & 2 \\
\hline Subtotal & 1 & 1 & 2 \\
\hline $\begin{array}{l}5.0 \text { - MATERIALS [Materiais] } \\
\text { science, technology, medicine materials [materiais em CES T e medic.] } \\
\text { bibliometrics, scientometrics, informetrics [bibliomet., cienciomet., } \\
\text { informetr.] }\end{array}$ & 3 & 1 & 4 \\
\hline Subtotal & 5 & 1 & 6 \\
\hline $\begin{array}{l}\text { 12.0 - BIBLIOGRAPHIC RECORDS [Registro Bibliográfico] } \\
\text { index language and systems [sistemas e linguagens de indexação] }\end{array}$ & 1 & - & 1 \\
\hline Subtotal & 1 & - & 1 \\
\hline $\begin{array}{l}15.0 \text { - READING [Leitura] } \\
\text { literacy [alfabetização] }\end{array}$ & 1 & - & 1 \\
\hline Subtotal & 1 & - & 1 \\
\hline $\begin{array}{l}\text { 19.0 - OTHER FRINGE SUBJECTS [Outros Assuntos Correlatos] } \\
\text { Literatura } \\
\text { meios de comunicação de massa } \\
\text { Semiologia }\end{array}$ & $\begin{array}{l}1 \\
1 \\
-\end{array}$ & $\dot{-}$ & $\begin{array}{l}1 \\
1 \\
1\end{array}$ \\
\hline Subtotal & 2 & 1 & 3 \\
\hline TOTAL & 10 & 3 & 13 \\
\hline
\end{tabular}

LEGENDA: D - Doutorado; M - Mestrado; T - Total

\section{CONSIDERACุÕES FINAIS}

A partir dos resultados obtidos na distribuição temporal da produção de dissertações/teses, é possível concluir que, mesmo com períodos de altos e baixos, a pós-graduação na ECA/USP na área de concentração ciência da informação e documentação manteve-se estável com tendência a crescimento. Acredita-se que a produção tenda a aumentar, pois o atual corpo docente do CBD, em sua maioria, possui os requisitos necessários para orientação e os demais encontram-se aptos ao credenciamento, caracterizando a consolidação de uma massa crítica.

O instrumento utilizado para a classificação temática das dissertações/teses atendeu aos objetivos desta pesquisa. Pode-se considerar que as dificuldades enfrentadas na classificação dos trabalhos e escolha dos termos são naturais da indexação e das características peculiares da produção analisada, que provavelmente diferem dos critérios utilizados pelo LISA na indexação de artigos. Na ausência de uma ferramenta considerada completa, sugere-se que sejam realizados trabalhos para a validação e adaptação de instrumentos de indexação disponíveis que sirvam de parâmetro para os diferentes estudos de análise temática da produção científica nacional em ciência da informação.

Ci. Inf., Brasília, v. 33, n. 2, p. 132-142, maio/ago. 2004
As temáticas abordadas na produção refletem as características da área de concentração e de suas linhas de pesquisa. Ressalta-se que as categorias que obtiveram pequeno número de trabalhos podem ser consideradas como indícios de novos rumos ou complementações das atuais investigações na pós-graduação do CBD, ou ainda temas de pouco interesse. Porém, é necessário verificar em pesquisas posteriores se as mesmas se mantém ou desaparecem. Observando as subcategorias, verifica-se grande diversidade de temas, o que não necessariamente significa dispersão, e sim interesses por diferentes aspectos e abordagens das categorias contempladas. De modo geral, as temáticas abordadas nas linhas de pesquisa estão condizentes aos objetivos das mesmas. No entanto, constatou-se que, em alguns casos, existem subcategorias que fogem do foco da linha, o que provavelmente está relacionado a fatores que influenciam a escolha pessoal do tema e, mais especificamente, às características acadêmicas e de pesquisa de determinados orientadores que fazem parte de cada linha. Seria interessante uma análise do perfil dos docentes e de sua produção científica em confronto com os temas das dissertações/ teses que orientam, como um dos possíveis subsídios para a avaliação das linhas de pesquisa desenvolvidas nos programas de pós-graduação em ciência da informação.

Artigo recebido em 16-06-2004 e aceito para publicação de 18 a 21/10/2004. 


\section{Fernanda Mendes Queiroz / Daisy Pires Noronha}

\section{REFERENCIAS}

ARAUJO, Eliany Alvarenga de; TENÓRIO, Jovana Karla Gomes; FARIAS, Simarle Nóbrega de. A produção do conhecimento na ciência da informação: análise das dissertações produzidas no curso de mestrado em CI$\mathrm{CMCl} / \mathrm{UFPb}$, no período de 1996/2001. In: ENCONTRO NACIONAL DE PESQUISA EM CIÊNCIA DA INFORMAÇÃO, 5., 2003, Belo Horizonte. Anais... Belo Horizonte: ANCIB, 2003. 1 CD-ROM.

EVANGELISTA, Rosana. Objetos de estudo das Dissertações do Mestrado em Biblioteconomia da PUC-Campinas: uma contribuição para o referencial teórico da área. 2002. 110f. Dissertação (Mestrado em Biblioteconomia e Ciência da Informação) - Faculdade de Biblioteconomia e Ciência da Informação, Pontifícia Universidade Católica de Campinas, Campinas, 2002.

GRACELLI, Aldemir; CASTRO, Cláudio de Moura. O desenvolvimento da pós-graduação no Brasil. Ciência e Cultura, v. 37, n. 7, p. 188-201, jul. 1985. Suplemento.

LIBRARY AND INFORMATION SCIENCE ABSTRACTS. Broad subject headings. London : Library Association, n. 1, Jan. 2003.

MACEDO, Neusa Dias de. Pesquisa em ciência da informação e biblioteconomia: questões de base; aplicações na pós-graduação; análise temática. Ciência da Informação, Brasília, v. 16, n. 2, p. 129. 144, jul./dez. 1987.

MACIAS-CHAPULA, Cesar A. O papel da informetria e da cienciometria e sua perspectiva nacional. Ciência da Informação, Brasília, v. 27, n. 2, p. 134-140, maio/ago.1998.

OLIVEIRA, Marlene de. Características das dissertações de mestrados produzidas no curso de mestrado em ciência da informação da UFPB. Informação EF Sociedade: Estudos, João Pessoa, v. 9, n. 2, 1999. Disponível em: < http://www.informacaoesociedade.ufpb.br/ 929922.html>. Acesso em: 16 abr. 2003.
PINTO, Virgílio Benjamin Noya. Pós-graduação em comunicação na USP: preservando a diversidade e a interdisciplinaridade. Intercom . Revista Brasileira de Comunicação, São Paulo, n. 59, p. 107-109, jul. 1988.

POBlACIÓN, Dinah Apparecida de Mello Aguiar. Pesquisa e PósGraduação em ciência da informação e biblioteconomia no Brasil: duas fases (1970/85 - 1986/92). In: ENCONTRO NACIONAL DOS CURSOS DE PÓS-GRADUAÇÃO EM CIÊNCIA DA INFORMAÇÃO E BiblioteCONOMIA, 12., 1992, São Paulo. Anais... São Paulo: ANCIB, 1993. p. 11-23.

SMIT, Johana W. A política governamental para a pós-graduação em ciência da informação no Brasil. Informação $\mathcal{E}$ Sociedade: Estudos, João Pessoa, v. 9, n. 2, 1999. Disponível em: <http:// www.informacaoesociedade.ufpb.br/929915.html>. Acesso em: 16 abr. 2003.

TEIXEIRA, Sonia Kazuo Sakai. Temática das dissertações defendidas no curso de mestrado em biblioteconomia e documentação da Universidade de Brasília. 1997. 135 f. Dissertação (Mestrado em Biblioteconomia) Faculdade de Estudos Sociais Aplicados, Universidade de Brasília, Brasília, 1997.

UNIVERSIDADE DE SÃO PAULO. Escola de Comunicações e Artes. Cursos de pós-graduação. São Paulo, [2002].

Núcleo de Produção Científica. Programas de Pós-graduação. Disponivel em: <http://www.eca.usp.br/nucleos/pc/PPG.htm>. Acesso em: 07 jul. 2003.

WITTER, Geraldina Porto; PÉCORA, Gláucia Maria Mollo. Temática das dissertações e teses em biblioteconomia e ciência da informação no Brasil. In: 1997. p. $77-86$. 